九州大学学術情報リポジトリ

Kyushu University Institutional Repository

Trogositidae, Languriidae, Tenebrionidae and Alleculidae from Korea (incl. Chejudo Is.) (Coleoptera)

Chujo, Michitaka

Lee, Chang Eon

https://doi.org/10.5109/2574

出版情報: ESAKIA. 34，pp.187-193，1994-03-24. Entomological Laboratory，Faculty of Agriculture, Kyushu University

バージョン :

権利関係 : 


\title{
Trogositidae, Languriidae, Tenebrionidae and Alleculidae from Korea (incl. Chejudo Is.)(Coleoptera) ${ }^{1,2)}$
}

\author{
Michitaka CHÔJO \\ Hikosan Biological Laboratory, Faculty of Agriculture, \\ Kyushu University, Hikosan, Fukuoka 824-07 \\ and \\ Chang Eon LeE \\ Department of Biology, College of Natural Science, Kyungpook \\ National University, Taegu, 702-701 Korea
}

\begin{abstract}
Two species of Trogositidae, five species of Tenebrionidae and five species of Alleculidae are newly added to the Korean coleopterous fauna.
\end{abstract}

\section{TROGOSITIDAE}

Leperininae

\section{Leperina squamulosa Gebler}

Leperina squamulosa Gebler, 1830, Ledebours Reise, 2: 97 (E. Siberia).

Spec. exam.: 1 ex. (light trap), Naelyeong-Ri, Samnae Myeon, Cheonla-buk do, S-Korea, 13. vii. 1991, M. T. Chûjô leg.; 1 ex., Kwang Nung, Pochon-gun, Kyonggi-do, Korea, 14-19. v. 1992, M. T. Chûjô leg.

Gen. distr.: Korea*; Japan (Hokkaido \& Honshu), E-N. China, Mongolia \& E. Siberia.

1) Results from the Korea-Japan Co-operative Science Program on "The Evolution and Biogeography of the Insects in the East Asia, No. 20.

2) Contribution from the Hikosan Biological Laboratory, Faculty of Agriculture, Kyushu University (Ser. 4. No. 11).

Asterisk shows the species which has hitherto been unrecorded from mainland of Korea and Chejudo Is. 


\section{Ostominae}

\section{Thymalus parviceps Lewis}

Thymalus parviceps Lewis, 1894, Ent. Monthl. Mag., (2) 5, 30:33-34 (Japan: Honshu).

Spec. exam.: 2 exs., Younsil, Mt. Hallasan, Chejudo, Korea, 24. vii. 1990, M. T. Chûjô leg.; 13 exs., Orimok, Mt. Hallasan, Chejudo, Korea, 27. vii. 1990, M. T. Chûjô leg.; 5 exs., JeongLyong Chy, SanNae Meon, NamWeon Gun, Jeong Ra Bug do, Korea, 28. ix. 1991, M. T. Chûjô leg.; 3 exs., Kwang Nung, Pochon-gun, Kyonggi-do, Korea, 14-19. v. 1992, M. T. Chûjô leg.; 1 ex., GamJong-ri, ChunChon-gun, KangWon-do, Korea, 21. v. 1992, M. T. Chûjô leg.; 1 ex., BougMyong-ri, ChunChon-gun, KangWon-do, Korea, 22-23. v. 1992, M. T. Chûjô leg.; 1 ex., BougMyong-ri, ChunChon-gun, KangWon-do, Korea, 21. vii. 1992, M. T. Chûjô leg.; 5 exs., Kwang Nung, Pochon-gun, Kyonggi-do, Korea, 16-19. vii. 1992, M. T. Chûjô leg.; 8 exs., YongshilOrimok, Mt. Halla, Chejudo, Korea, 23. vii. 1993, M. T. Chûjô leg.; 28 exs., Kwanum Temple, Chejudo, Korea, 2.5. vii. 1993, M. T. Chûjô leg.

Gen. distr.: Korea*(incl. Chejudo Is.*); Japan (Hokkaido, Honshu, Shikoku \& Kyushu) \& Sakhalien.

\section{LANGURIIDAE}

\section{Tetralanguria collaris (Crotch)}

Pachylanguria collaris Crotch, 1876, Cist. Ent., 1: 377 (India).

Spec. exam.: 9 exs., Kwang Nung, Pochon-gun, Kyonggi-do, Korea, 17. v. 1992, K. Morimoto leg.

Gen. distr.: Korea; Japan (Ryukyus), Formosa, China, India, Indochina \& Tibet.

\section{TENEBRIONIDAE}

Pedinini

\section{Pedinus (Blindus) strigosus Faldermann}

Pedinus strigosus Faldermann, 1835, Mém. Acad. St. Pétersb. sav. etr., 2: 410 (China).

Spec. exam.: 1 ex., SamJeong-li, MaCheong-meon, Gyeongsam Nam-do, Korea, 11. v. 1991, S. Nomura leg.; 1 ex., SamJeong-li, MaCheong-meon, GyeongSam Nam-do, Korea, 1.5. v. 1991, S. Nomura leg.

Gen. distr.: Korea proper; Japan (Tsushima Is.), Siberia, Mongolia \& N. China. 


\section{Gonocephalum coriaceum Motschulsky}

Gonocephalum coriaceum Motschulsky, 1857, Étud. Ent., 6: 34 (Japan).

Spec. exam.: 2 exs., Dae Chong, Boryeong Gun, Korea, 8 \& 9. vi. 1983, K. Morimoto leg.

Gen. distr.: Korea proper; Japan proper, Siberia, China, Formosa, Nepal, S-E. Asia, Micronesia, Samoa Is., Irian Jaya, Africa, Madagascar \& Australia.

\section{Diaperini}

\section{Platydema recticome Lewis}

Platydema recticorne Lewis, 1894, Ann. Mag. Nat. Hist., [6],13(77): 394-395 (Japan: Honshu).

Spec. exam.: 10 exs., Kwanum Temple, Chejudo City, Chejudo, Korea, 25. vii. 1993, M. T. Chûjô leg.

Gen. distr.: Korea (Chejudo Is.*); Japan (Honshu, Shikoku, Kyushu, Tanegashima Is., Yakushima Is., Nakanoshima Is. \& Iriomotejima Is.).

\section{Platydema subfascia subfascia (Walker)}

Alphitophagus subfascia Walker, 1858, Ann. Mag. Nat. Hist., [3],2(10): 284 (Ceylon).

Spec. exam.: 2 exs., Kwangnyong-ri, Aewol-up, Chejudo Is., Korea, 22. vii. 1993, M. T. Chûjo leg.

Gen. distr.: Korea (incl. Chejudo Is.*); Japan proper (excl. Yaeyama Isls.), Formosa, Indochina, Burma, India, Sri Lanka, Borneo, Sumatra, Malacca, Mentawai, Java \& Madagascar).

\section{Helopini}

\section{Tarpela cordicollis (Marseul)}

Helops cordicollis Marseul, 1876, Ann. Soc. Ent. France, (5) 6: 141-142 (Japan: Kyushu).

Spec. exam.: 1 ex., HongCheon, GanWeon do, Korea, 18 \& 19. v. 1984, K. Morimoto leg.

Gen. distr.: Korea* \&Japan (Honshu, Shikoku \& Kyushu).

\section{Heterotarsini}

\section{Anaedus mroczkowskii Kaszab}

Anaedus mroczkowskii Kaszab, 1968, Ann. Zool. Warszawa, 26(2):10-11(N. Korea).

Spec. exam.: 1 ex., Kwang Nung, Pochon-gun, Kyonggi-do, Korea, 14-19. v. 1992, S. Nomura leg.; 1 ex., BougMyong-ri, ChunChon-gun, KwangWeon-do, Korea, 22-23. v. 1992, S. Nomura leg.

Gen. distr.: Korea*(incl. Chejudo Is.) \& N. Korea.

\section{Luprops cribrifrons Marseul}

Luprops cribrifrons Marseul, 1876, Ann. Soc. Ent. France, (5) 6: 125-126 (Japan: Kyushu \& 
Honshu).

Spec. exam.: 8 exs., KwangNyong-ri, Aewol-up, Cheju City, Chejudo, Korea, 22. vii. 1993, M. T. Chûjô leg.

Gen. distr.: Korea (Chejudo Is*); Japan (Honshu, Shikoku, Kyushu, Tsushima Is. \& Goto Isls.), Formosa \& Indochina.

\section{ALLECULIDAE}

\section{Alleculinae}

\section{Allecula melanaria Mäklin}

Allecula melanaria Mäklin, 1875, Acta Soc. Fennica, 10: 669(519) (Japan).

Spec. exam.: 8 exs., NaeLyon Ri, SamNae Meon, NamWeong Gun, JeongRa Bug do, Korea, 26. ix. 1991, M. T. Chûjô leg.; 3 exs., SamJeong Ri, MaCheong Meon, Ham Yang Gun, GyeongSang Nam do, Korea, 29. ix. 1991, M. T. Chûjô leg.

Gen. distr.: Korea*; Japan (Hokkaido, Honshu, Shikoku, Kyushu \& Ishigakijima Is.).

\section{Allecula noctivaga Lewis}

Allecula noctivaga Lewis, 1895, Ann. Mag. Nat. Hist., [6],15(87): 251 (Japan: Honshu).

Spec. exam.: 1 ex., BougMyong-ri, ChunChon-gun, KangWeon-do, Korea, 23. vii. 1992, M. T. Chûjô leg.

Gen. distr.: Korea* \& Japan (Honshu \& Tsushima Is.).

\section{Borboresthes acicularis (Marseul)}

Alleculaacicularis Marseul, 1876, Ann. Soc. Ent. France, (5) 6: 325-326 (Japan: Honshu).

Spec. exam.: 2 exs.(malaise trap), Mt. Hallasan, Yongshil, Chejudo Is., Korea, 24-27. vii. 1990, K. Morimoto leg.; 1 ex., Naelyong Ri, Cheonla-buk do, S-Korea, 13. vii. 1991, M. T.Chûjô leg.; 1 ex., DooRyu Dong, JooChun Meon, Sam-Chung Gun, Gyeong Sang Nam do, Korea, 25. ix. 1991, M. T. Chûjjô leg.; 2 exs., Kwang Nung, Pochon-gun, Kyonggi-do, Korea, 16-19. vii. 1992, M. T. Chûjô leg.; 2 exs., Boug-Myong-ri, ChunChon-gun, KangWeon-do, Korea, 21. vi. 1992, M. T. Chûjô leg.

Gen. distr.: Korea*(Chejudo Is.*) \& Japan (Honshu, Shikoku, Kyushu \& Tsushima Is.).

\section{Hymenalia rujipennis (Marseul)}

Cistela(Gonodera) rufipennis Marseul, 1876, Ann. Soc. Ent. France, (5) 6: 328-329 (Japan: Honshu \& Kyushu).

Spec. exam.: 1 ex., Naelyong Ri, Samnae Myeon, Cheonla-buk do, S-Korea, 13. vii. 1991, M. T. Chûjô leg.; 2 exs., Sam-jeong Ri, MaCheon Myeon, Cheonla-buk-do, S-Korea, 14. vii. 1991, M. T. Chûjô leg.

Gen. distr.: Korea*; Japan (Honshu, Shikoku \& Kyushu), Siberia \& Formosa. 


\section{Hymenalia unicolor Nakane}

Hymenalia unicolor Nakane, 1963, Fragm. Col., Tokyo, (7): 30 (Japan: Honshu).

Spec. exam.: 2 exs., Naelyong Ri, Samnae Myeon, Cheonla-buk do, S-Korea, 14. vii. 1991, M. T. Chûjô leg.; 5 exs., Samjeong Ri, MaCheon Myeon, Cheonla-buk do, S-Korea, 14. vii. 1991, M. T. Chûjô leg.

Gen. distr.: Korea* \& Japan (Hokkaido, Honshu, Shikoku, Kyushu \& Tsushima Is.).

\section{ACKNOWLEDGEMENTS}

We are very greateful to Director General Jae Myeong Jo'of Forest Research Institution, Korea and Director Seung Kul Park of Chung-bu Forest Experiment Station, Korea for their verious help to our collecting trip under the Korea-Japan Co-operative Science Program on "The Evolution and Biogeography of the Insects on the East Asia. And also, we express our cordial thanks to Prof. Dr. Chang Young Lee and Dr. Jong Kuk Kim, Kwangweon National University, Korea for their miscellaneous help through our collecting trip.

We wish to express our appreciation to Prof. Dr. K. Morimoto, Entomological Laboratory of Kyushu University for his continual kind and valuable guidance. And we are thankful to Assoc. Prof. Dr. 0. Tadauchi and Assist. Prof. S. Nomura of Entomological Laboratory of Kyushu University for their various co-operation for this work.

\section{REFERENCES}

Cho, P. S., 1957. A Systematic Catalogue of Korean Coleoptera. Humanities and Sciences, Korea University 2: 173-326 (In Korean).

1963,4. Insects of Quelpeart Island (Cheju-do). Humanities and Sciences, Korea University, 6: 159-242 (In Korean).

Chûjô, M. T. et al., 1992. Nitidulidae from Chejudo Island. Esakia, Kyushu Univ. Publ. Ent., (32): 19-24, 9 figs.

- 1992. Tenebrionidae from Chejudo Island, Korea (Insecta Coleoptera). ibid., 31-46, 11 figs. 1993. Korean Tenebrionidae (Insecta, Coleoptera). Esakia, Kyushu Univ. Publ. Ent., (33): 109-122, 12 figs.

Crotch, G. R., 1876. A rvision of the coleopterous family Erotylidae. Cistula Ent., 1: 377-572.

Kaszab, Z., 1952. Die indomalaischen und ostasiatischen Arten der Gattung Gonocephalum Solier (Coleoptera, Tenebrionidae). Ent.Arb. Mus. G. Frey, 3 (2): 1-688,511 figs.

- 1968. Tenebrionidae und Meloidae (Coleoptera) aus Nord-Korea, gesammelt von M. Mroczkowski und A. Riedel im Jahre 1965. Ann. Zool., Warszawa, 26 (2): 7-14.

Lee, Y. I. et al., 1985. Insect fauna of Mt. Halla. Rep. Acad. Surv., Hallasan (Mt.) Nat. Pres., Cheju- 
do, Korea : 351-455.

Lewis, G., 1894. On the Tenebrionidae of Japan. Ann. Mag. Nat. Hist., [6], 13 (77): 377-484, 1 pl., 16 figs.

1894. On new species of Trogositidae from Japan. Ent. Monthl. Mag., [2] 5, 30: 32-34.

1895. On the Cistelidae and other Heteromerous species of Japan. Ann. Mag. Nat. Hist., [6], 15 (87): 250-279, 1 pl. 12 figs.

Marseul, M. S.-A., 1876. Coleopteres du Japon recueillis par H. George Lewis. Ann. Soc. Ent. France, (5) 6: 219-486.

Medvedev, G. S., 1992. Key to the identification of insects of the Soviet Far East. III. Coleoptera. 704 pp., 314 figs.

Nakane, T., 1963. New or little known Coleoptera from Japan and its adjacent regions. Fragm. Col., Tokyo, (7): 30.

Okamoto, H., 1924. The insect fauna of Quelpart Island (Saishu-to). Bull. Algr: Exper: Stat., Gov.Gener. Chosen, 1 (2): 233pp., 10 pls.

Seok, D. M., 1970. The Insect Fauna of the Is. Quelpart. Boo Sin Sai K. K., Scoul, 186 pp.

\section{Explanation of Plate I}

1. Leperina squamulosa Gebler . . Length: $13.7 \mathrm{~mm}$.

2. Thymalus parviceps Lewis . . L.: $6.1 \mathrm{~mm}$.

3. Tetralanguria collaris (Crotch) . L.: 14.6mm.

4. Platydema recticorne Lewis .. L.: $4.4 \mathrm{~mm}$.

5. Platydema subfascia subfascia (Walker) . L.: $4.4 \mathrm{~mm}$.

6. Allecula melanaria Mäklin . L.: $10.2 \mathrm{~mm}$.

7. Allecula noctivaga Lewis . . L.: $9.8 \mathrm{~mm}$.

8. Borboresthes acicularis (Marseul) . L.: $6.6 \mathrm{~mm}$.

9. Hymenalia rufipennis (Marseul) . L.:6.1mm.

10. Hymenalia unicolor Nakane . , L.: 6.8mm.

11. Luprops cribrifrons Marseul L.: $8.0 \mathrm{~mm}$. 


\section{PLATE 1}
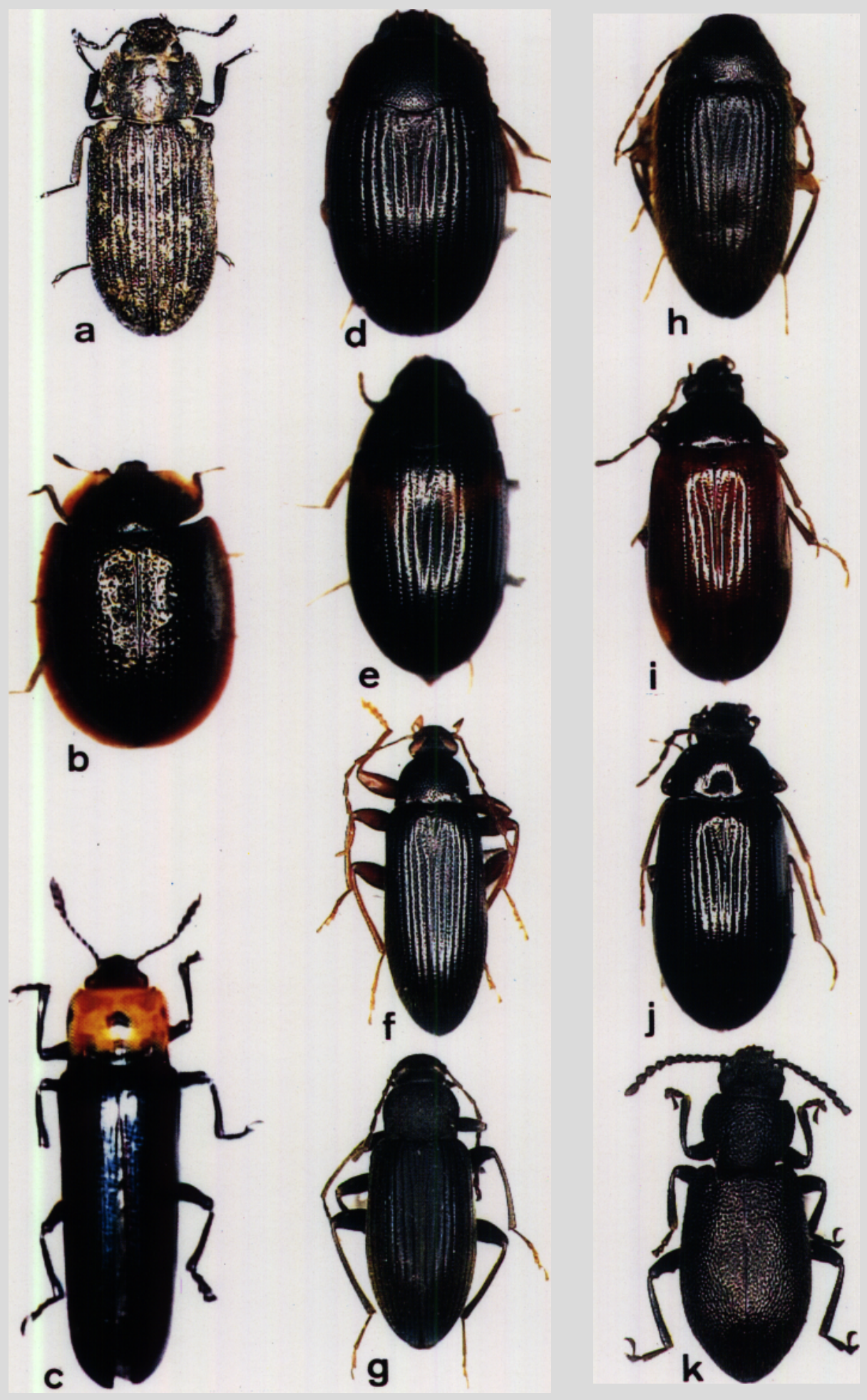